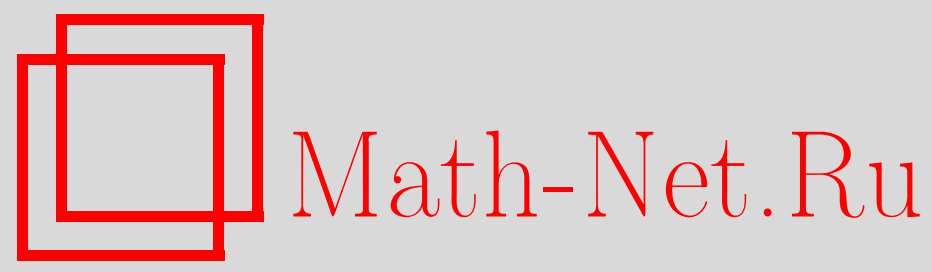

А. В. Лебедев, Общая схема максимумов сумм независимых случайных величин и ее приложения, Матем. заметкu, 2005, том 77, выпуск 4, 544-550

DOI: https://doi.org/10.4213/mzm2516

Использование Общероссийского математического портала Math-Net.Ru подразумевает, что вы прочитали и согласны с пользовательским соглашением http://www . mathnet.ru/rus/agreement

Параметры загрузки:

IP : 54.164 .48 .24

26 апреля 2023 г., 05:54:58 


\section{ОБЩАЯ СХЕМА МАКСИМУМОВ СУММ НЕЗАВИСИМЫХ СЛУЧАЙНЫХ ВЕЛИЧИН И ЕЕ ПРИЛОЖЕНИЯ}

\section{А.В. Лебедев}

Вводится общая схема максимумов сумм независимых случайных величин. Доказана теорема о сходимости максимумов по вероятности. Рассмотрены ее приложения для больших скачков случайных блужданий и экстремумов полей дробового шума в случае правильно меняющихся хвостов. Получены невырожденные предельные законы.

Библиограффия: 9 названий.

1. Введение. Простейшая схема максимумов сумм имеет вид

$$
\zeta_{m, n}=\max _{1 \leqslant i \leqslant m} \sum_{j=1}^{n} \xi_{i, j},
$$

где случайные величины $\xi_{i, j}, i \geqslant 1, j \geqslant 1$, независимы и одинаково распределены.

Асимптотическое поведение величин $\zeta_{m, n}$ при $m, n \rightarrow \infty$ впервые изучалось в [1]. Некоторые новые результаты типа невырожденных предельньх законов были получены в [2]. Модель (1) может описьвать, например, время выполнения однотипных работ, проводимых параллельно, если каждая из них делится на множество фаз [3], а также некоторые нормы матриц из независимых случайных элементов [2] и др.

Далее будет построена общая схема, допускающая случайные и пересекающиеся множества индексов слагаемых сумм при произвольном числе этих сумм, по которым берется максимум. Будет доказана одна предельная теорема для случая неотрицательных слагаемых и показано, как она может быть применена к изучению больших скачков случайных блужданий [4] и экстремумов полей дробового шума [5] с правильно меняющимися хвостами амплитуд.

2. Описание модели и основные результаты. Пусть задан случайный процесс $\Upsilon=\{\Upsilon(t), t \in T\}$, значениями которого являются конечные классы конечных подмножеств $\mathbf{N}$, а параметрическое множество $T$ содержит бесконечно много элементов.

Во избежание проблем в определении процесса с нечисловьми значениями заметим, что множество конечных классов конечных подмножеств $\mathbf{N}$ счетно, так что можно установить взаимно однозначное соответствие между $\Upsilon(t)$ и некоторьм случайным процессом $\Upsilon^{*}(t)$ со значениями в $\mathbf{N}$.

Работа выполнена при поддержке Российского фонда фундаментальных исследований, гранты № 03-01-00724 и № 04-01-00700, и программы “Ведущие научные школы”, грант № НШ 1758.2003.1. 
Пусть задано семейство $\Xi=\left\{\xi_{i, t}, i \in \mathbf{N}, t \in T\right\}$ неотрищательных случайных величин, независимых и одинаково распределенных при любом фиксированном значении параметра $t$.

Полагаем, что $\Upsilon$ и $\Xi$ независимы.

Для любых $A \subset \mathbf{N}, t \in T$ обозначим максимум набора случайных величин $\left\{\xi_{i, t}, i \in A\right\}$ через $M_{t}(A), r$-й максимум (т.е. число, стоящее $r$-м с конца в вариационном ряду) через $M_{t}^{(r)}$, сумму через $S_{t}(A)$.

Пусть $U(t)=\bigcup_{A \in \Upsilon(t)} A$.

Введем случайные процессы, порожденные $\Upsilon$ и $\Xi$ :

$$
\begin{gathered}
\zeta(t)=\sup _{A \in \Upsilon(t)} S_{t}(A), \quad \kappa(t)=\sup _{A \in \Upsilon(t)}|A|, \quad \nu(t)=|U(t)|, \\
\mu_{1}(t)=M_{t}(U(t)), \quad \mu_{r}(t)=M_{t}^{(r)}(U(t)),
\end{gathered}
$$

где через $|A|$ обозначается число элементов множества $A$.

Предполагается, что $\nu(t)<\infty$ почти наверное (п.н.) при всех $t \in T$, откуда следует конечность п.н. всех процессов, введенных выше.

Нас интересует предельное поведение $\zeta(t)$ при $t \rightarrow \infty$.

Если $T=\mathbf{N}$ или $T=\mathbb{R}_{+}$, используем стандартное понятие предела действительной функции $f(t)$ при $t \rightarrow \infty$. В других случаях (например, когда процесс параметризован ограниченньми измеримыми областями в конечномерном пространстве) понятие такого предела вводится дополнительно.

Пусть существует случайньй процесс $\rho(t)$ со значениями в $\mathbb{Z}_{+}$, измеримьй относительно $\Upsilon$, такой, что $\rho(t) \geqslant 1$ при $\nu(t) \geqslant 1, \rho(t) \leqslant \nu(t)$ п.н. при всех $t \in T$.

Обозначим через $\pi(t)$ вероятность того, что для множества $B$, равновероятно выбранного среди всех подмножеств $U(t)$, состоящих из $\rho(t)$ элементов, имеет место

$$
\sup _{A \in \Upsilon(t)}|A \cap B|>1
$$

ТЕОРЕМа 1. Пусть выполнены условия

$$
\begin{gathered}
(\kappa(t)-1) \frac{\mu_{\rho(t)}(t)}{\mu_{1}(t)} \stackrel{\stackrel{\mathrm{P}}{\rightarrow} 0, \quad t \rightarrow \infty,}{\pi(t) \rightarrow 0, \quad t \rightarrow \infty .}
\end{gathered}
$$

Toгдa

$$
\frac{\zeta(t)}{\mu_{1}(t)} \stackrel{\mathrm{P}}{\rightarrow} 1, \quad t \rightarrow \infty
$$

ДокАЗАТЕЛьство. Заметим, что по определению $\zeta(t) \geqslant \mu_{1}(t)$ и, следовательно, нужно получить лишь оценку для $\zeta(t)$ сверху.

При каждом $t \in T$ подмножество $I(t)$ индексов $\rho(t)$ наибольших случайных величин в наборе $\left\{\xi_{i, t}, i \in U(t)\right\}$ оказьвается выбранным равновероятно среди всех множеств такого размера в силу независимости $\Upsilon$ и $\Xi$. Таким образом, по (3) вероятность того, 
что хоть в одной сумме $S_{t}(A)$ окажется более одного слагаемого из $\rho(t)$ наибольших, стремится к нулю.

На множестве $\left\{\omega: \sup _{A \in \Upsilon(t)}|A \cap I(t)| \leqslant 1\right\}$, вероятность которого стремится к единище, имеет место равномерная оценка $S_{t}(A) \leqslant \mu_{1}(t)+(\kappa(t)-1) \mu_{\rho(t)}(t)$, откуда следует

$$
\zeta(t) \leqslant \mu_{1}(t)+(\kappa(t)-1) \mu_{\rho(t)}(t) .
$$

В силу (2) отсюда получаем (4).

СлЕДСТВИЕ 1. Если $\kappa(t) \stackrel{\mathrm{P}}{\rightarrow} 1$ nрu $t \rightarrow \infty$, mo $(2)$ выполняется $п р и$ любом $\rho(t)$.

Это очевидно, поскольку $\mu_{\rho(t)}(t) \leqslant \mu_{1}(t)$.

Необходимо прояснить вопрос, когда еще условия теоремы 1 могут выполняться одновременно. Для этого понадобятся следующие утверждения о свойствах порядковых статистик в случае правильно меняющихся хвостов распределений.

3. Некоторые свойства порядковых статистик. Пусть $X_{n}, n \geqslant 1$, независимы и имеют распределение $F(x)$ с правильно меняющимся хвостом $\bar{F}(x) \sim x^{-\alpha} L(x)$, $x \rightarrow \infty, \alpha>0, L(x)$ - медленно меняющаяся функция [6, с. 317]. Обозначим максимум $X_{1}, \ldots, X_{n}$ через $\widetilde{X}_{n}$ и $r$-й максимум через $\widetilde{X}_{n}^{(r)}$.

Введем функцию $u(y)>0, y \geqslant 0$, такую, что

$$
y \bar{F}(u(y)) \rightarrow 1, \quad y \rightarrow \infty
$$

Можно взять, например, $u(y)=\sup \{x>1: y \bar{F}(x) \geqslant 1\}$; тогда в силу правильного изменения хвоста будет выполняться (5).

Из определения (5) следует, что $u\left(y^{\prime}\right) \sim u\left(y^{\prime \prime}\right)$ при $y^{\prime} \sim y^{\prime \prime} \rightarrow \infty$ и

$$
u(y) \sim y^{1 / \alpha+o(1)}, \quad y \rightarrow \infty .
$$

Заметим, что для максимумов случайных величин $X_{n}, n \geqslant 1$, имеет место невырожденньй предельньй закон [6, с. 319], [7, с. 28]:

$$
\lim _{n \rightarrow \infty} \mathrm{P}\left(\frac{\tilde{X}_{n}}{u(n)} \leqslant x\right)=\Phi_{\alpha}(x)
$$

где $\Phi_{\alpha}(x)=\exp \left\{-x^{-\alpha}\right\}, x>0$ (распределение $\Phi$ реше).

ЛЕмма 1. Пусть $r_{n} \sim n^{\gamma}, \gamma \in(0,1)$. Тогда

$$
\frac{\widetilde{X}_{n}^{\left(r_{n}\right)}}{u\left(n^{1-\gamma}\right)} \stackrel{\mathrm{P}}{\rightarrow} 1, \quad n \rightarrow \infty
$$


ДоКАЗАТЕЛЬСТво. Обозначим $c_{n}=u\left(n^{1-\gamma}\right)$. Пусть $x>0$ и

$$
S_{n}=\sum_{k=1}^{n} \mathrm{I}\left(X_{k}>x c_{n}\right)
$$

где I $(A)$ - индикатор события $A$. Каждое слагаемое в $S_{n}$ равно единице с вероятностью $p_{n}=\bar{F}\left(x c_{n}\right) \sim x^{-\alpha} / n^{1-\gamma}, n \rightarrow \infty$, независимо от других. Положим $Q_{n}=S_{n} / r_{n}$; тогда

$$
\mathrm{M} Q_{n}=n \frac{p_{n}}{r_{n}} \rightarrow x^{-\alpha}, \quad \mathrm{D} Q_{n}=n \frac{p_{n}\left(1-p_{n}\right)}{r_{n}^{2}} \rightarrow 0,
$$

откуда следует $Q_{n} \stackrel{\mathrm{P}}{\rightarrow} x^{-\alpha}, n \rightarrow \infty$. Воспользуемся соотношением [7, с. 45]

$$
\mathrm{P}\left(\widetilde{X}_{n}^{\left(r_{n}\right)} \leqslant x c_{n}\right)=\mathrm{P}\left(S_{n}<r_{n}\right)=\mathrm{P}\left(Q_{n}<1\right) .
$$

Рассмотрев случаи $x>1$ и $x<1$, получаем утверждение леммы 1 .

СлЕДСТвиЕ 2. Пусть $0<\delta<\gamma / \alpha$. Тогда

$$
n^{\delta} \frac{\widetilde{X}_{n}^{\left(r_{n}\right)}}{\widetilde{X}_{n}} \stackrel{\mathrm{P}}{\rightarrow} 0, \quad n \rightarrow \infty .
$$

ДоКАЗАТЕЛЬСТво. Имеем

$$
n^{\delta} \frac{\widetilde{X}_{n}^{\left(r_{n}\right)}}{\widetilde{X}_{n}}=n^{\delta}\left(\frac{\widetilde{X}_{n}^{\left(r_{n}\right)} / u\left(n^{1-\gamma}\right)}{\widetilde{X}_{n} / u(n)}\right) \frac{u\left(n^{1-\gamma}\right)}{u(n)} \stackrel{\mathrm{P}}{\rightarrow} 0
$$

с учетом (6)-(8).

Далее всюду будем предполагать, что все случайные величины из $\Xi$ имеют одинаковое распределение $F$, удовлетворяюшее условиям

$$
F(-0)=0, \quad \bar{F}(x) \sim x^{-\alpha} L(x), \quad x \rightarrow \infty .
$$

Тогда пары $\left(\mu_{\rho(t)}(t), \mu_{1}(t)\right)$ при условиях $\rho(t)=r_{n}, \nu(t)=n$ распределены так же, как $\left(\widetilde{X}_{n}^{\left(r_{n}\right)}, \widetilde{X}_{n}\right)$, что открывает возможность применения теоремы 1.

4. Пример: большие скачки случайных блужданий. Пусть заданы последовательности независимых одинаково распределенных случайных величин $\eta_{n}$ и натуральных чисел $l_{n}, n \geqslant 1$. Определим

$$
Z_{n}=\max _{0 \leqslant k \leqslant n} \sum_{i=k+1}^{k+l_{n}} \eta_{i} .
$$

Асимптотическое поведение таких величин (статистик Эрдёша-Реньи) при $n \rightarrow \infty$, $l_{n} \rightarrow \infty$, изучалось, например, в [4]. Получены невырожденные предельные законы при $l_{n} \sim \ln n$ и т.п. Отмечены приложения модели в математической генетике (анализ ДНК).

В [4] предполагалось, что для слагаемых вьполнено правостороннее условие Краме$\mathrm{pa}$, т.е. $\mathrm{Me}^{h \eta_{1}}<\infty$ для некоторого $h>0$. Мы рассматриваем случай правильно меняюшихся хвостов, когда это условие не вьполняется.

Приведем задачу к виду, подходящему для применения теоремы 1 . В качестве параметра возьмем $n$, соответственно $T=\mathbf{N}$. Определим $\Xi$ через $\xi_{i, n} \equiv \eta_{i}$. Пусть $\Upsilon(n)$ состоит из множеств $A_{i, n}=\left\{i, \ldots, i+l_{n}-1\right\}, 1 \leqslant i \leqslant n+1$. Тогда $\zeta(n)=Z_{n}$, $\nu(n)=n+l_{n}, \kappa(n)=l_{n}, \mu_{1}(n)=\max _{1 \leqslant i \leqslant n+l_{n}} \eta_{i}$. 
Tеорема 2. Пусть $l_{n}=O\left(n^{\delta}\right), 0<\delta<1 /(1+2 \alpha)$. Тогда при любих $x>0$ верно

$$
\lim _{n \rightarrow \infty} \mathrm{P}\left(\frac{Z_{n}}{u(n)} \leqslant x\right)=\Phi_{\alpha}(x)
$$

где и(у) удовлетворяет (5).

ДокАЗАТЕЛЬСТВо. Положим $\rho(n)=\left[n^{\gamma}\right], \gamma=\alpha /(1+2 \alpha)$. Из комбинаторных соображений

$$
\pi(n) \leqslant \frac{\rho(n)(\rho(n)-1) l_{n}}{n+l_{n}-1} \sim n^{2 \gamma+\delta-1} \rightarrow 0, \quad n \rightarrow \infty,
$$

так что (3) выполнено. Поскольку $\kappa(n)=O\left(n^{\delta}\right), 0<\delta<\gamma / \alpha$, по следствию 2 также верно (2).

Таким образом, по теореме 1 имеем $\zeta(n) / \mu_{1}(n) \stackrel{\mathrm{P}}{\rightarrow} 1$. С учетом $(7)$ отсюда следует утверждение теоремы 2 , поскольку $u\left(n+l_{n}\right) \sim u(n), n \rightarrow \infty$.

Заметим, что произвольный сдвиг (в том числе, центрирование) $\eta_{n}$ не влияет на предельный результат. Действительно, пусть $\eta_{n}^{*}=\eta_{n}+a$; тогда $Z_{n}^{*}=Z_{n}+a l_{n}$, но $l_{n}=o(u(n)), n \rightarrow \infty$, так что теорема 2 сохраняет силу.

5. Пример: экстремумы полей дробового шума. Покажем, как можно применить полученные результаты к исследованию супремумов дробового шума в случае конечного радиуса влияния и правильно меняющихся хвостов распределений амплитуд [5]. Напомним эту модель (в других обозначениях), попутно обобщая ее.

Рассмотрим евклидово пространство $E=\mathbb{R}^{d}, d \geqslant 1$, и пусть $\Sigma=\left\{\sigma_{k}\right\}$ - стационарное пуассоновское точечное поле в $E$ интенсивности $\lambda>0$. Определим случайное поле

$$
\psi(s)=\bigoplus_{k} h_{k}\left(s-\sigma_{k}\right), \quad s \in E
$$

где $h_{k}$ - независимые одинаково распределенные измеримые вполне сепарабельные неотрицательные случайные функции на $E$, не зависящие от $\Sigma$, и $\oplus$ - коммутативная и ассоциативная бинарная операция такая, что $\max \{x, y\} \leqslant x \oplus y \leqslant x+y$ для любых $x, y \geqslant 0$.

Величины $h_{k}(0)$ назовем амплитудами, а их распределение обозначим через $F_{0}$. Предположим, что функции влияния удовлетворяют следуюшим условиям:

1) $h_{k}(s) \leqslant h_{k}(0)$ при всех $s \in E$;

2) $h_{k}(s)=0$ при $\|s\|>1$.

Имеется в виду, что 1), 2) вьполняются п.н. для всех $k$.

Сделанные предположения обеспечивают существование поля дробового шума $\psi(s)$ в смысле абсолютной сходимости (10) п.н., поскольку в каждой точке число слагаемых конечно п.н.

Определим теперь для произвольной ограниченной измеримой области $G$ супремум поля $\psi(s)$ по ней:

$$
\varphi(G)=\sup _{s \in G} \psi(s)
$$

Поле $\psi(s)$ оказывается вполне сепарабельным по построению, так что $\varphi(G)$ является случайной величиной. 
Нашей задачей будет применить к $\varphi(G)$ теорему 1 (при $G \rightarrow \infty$ ), построив некоторые $\Upsilon=\{\Upsilon(G)\}$ и $\Xi=\left\{\xi_{n, G}\right\}$.

Положим $\xi_{n, G} \equiv h_{n}(0)$. Пусть $\Upsilon(G)$ состоит из всех множеств вида $A_{s}=\left\{k:\left\|s-\sigma_{k}\right\|\right.$ $\leqslant 1\}, s \in G$, среди которых число различных конечно п.н. Тогда $U(G)=\left\{k: \sigma_{k} \in G_{1}\right\}$, где $G_{1}$ есть объединение области $G$ с ее единичной окрестностью. Получаем $\varphi(G) \leqslant$ $\zeta(G)$.

Обозначим меру Лебега области $G$ через $\langle G\rangle$ (во избежание путанищы с ранее введенньм обозначением числа элементов множества). Далее предполагаем, что $G$ растет таким образом, что $\left\langle G_{1}\right\rangle /\langle G\rangle \rightarrow 1$ и $\langle G\rangle \rightarrow \infty$. Для этого достаточно, например, чтобы $G \rightarrow \infty$ в смысле Ван Хова [5], [8, с. 30].

ЛЕмма 2. Для выполнения (3) в данной модели достаточно, чтобы

$$
\mathrm{M} \rho^{2}(G)=o(\langle G\rangle), \quad G \rightarrow \infty
$$

ДокАЗАТЕЛЬСтво. Выберем равновероятно множество $B$ из $\rho(G)$ элементов $U(G)$. Пусть $C=\left\{\sigma_{k}: k \in B\right\}$. В силу пуассоновости точечного поля $\Sigma$ точки $C$ независимо и равномерно распределены в $G_{1}$ при известных фиксированных $\rho(G), \nu(G)$. Обозначим

$$
\pi_{r, m}(G)=\mathrm{P}\left(\sup _{A \in \Upsilon(G)}|A \cap B|>1 \mid \rho(G)=r, \nu(G)=m\right) .
$$

Для того чтобы в единичную окрестность некоторой точки $s \in G$ попадало хотя бы две точки $C$, необходимо, чтобы расстояние между этими точками было не более 2 . Следовательно,

$$
\pi_{r, m}(G) \leqslant \frac{r(r-1)}{2} \frac{V_{2}}{\left\langle G_{1}\right\rangle},
$$

где $V_{2}$ - объем шара радиуса 2 в $\mathbb{R}^{d}$. Поскольку $\pi(G)=\mathrm{M} \pi_{\rho(G), \nu\left(G_{1}\right)}(G)$, отсюда следует утверждение леммы 2.

Пусть амплитуды дробового шума имеют распределение $F_{0}=F$.

Теорема 3. При любых $\alpha>0, x>0$ верно

$$
\lim _{G \rightarrow \infty} \mathrm{P}\left(\frac{\varphi(G)}{u(\lambda\langle G\rangle)} \leqslant x\right)=\Phi_{\alpha}(x) .
$$

ДоКАЗАТЕЛЬСТВо. Положим $\rho(G)=\left[\nu(G)^{\gamma}\right], 0<\gamma<1 / 2$; тогда

$$
\mathrm{M} \rho^{2}(G) \leqslant(\lambda\langle G\rangle)^{2 \gamma}=o(\langle G\rangle)
$$

и по лемме 2 выполнено (3).

Используем тот факт, что $\nu(G) \sim \lambda\left\langle G_{1}\right\rangle \sim \lambda\langle G\rangle$ п.н. при $G \rightarrow \infty$.

Заметим, что согласно теореме 1 из [9] в данном случае п.н. верно

$$
\kappa(G) \sim \frac{\ln \langle G\rangle}{\ln \ln \langle G\rangle}, \quad G \rightarrow \infty
$$


так что $\kappa(G)=o\left(\langle G\rangle^{\varepsilon}\right)=o\left(\nu(G)^{\varepsilon}\right)$ для любого $\varepsilon>0$, откуда по следствию 2 получаeм (2).

Таким образом, по теореме 1 имеем

$$
\frac{\zeta(G)}{\mu_{1}(G)} \stackrel{\mathrm{P}}{\rightarrow} 1, \quad G \rightarrow \infty .
$$

Заметим, что $\mu_{1}(G)$ распределено так же, как и $\widetilde{X}_{\nu(G)}$, так что с помощью (7) и асимптотики $u(\nu(G)) \sim u(\lambda\langle G\rangle)$ п.н. получаем

$$
\lim _{G \rightarrow \infty} \mathrm{P}\left(\frac{\mu_{1}(G)}{u(\lambda\langle G\rangle)} \leqslant x\right)=\Phi_{\alpha}(x),
$$

откуда с учетом (11) следует

$$
\lim _{G \rightarrow \infty} \mathrm{P}\left(\frac{\zeta(G)}{u(\lambda\langle G\rangle)} \leqslant x\right)=\Phi_{\alpha}(x) .
$$

Обозначим $\varphi_{0}(G)=\sup \left\{h_{k}(0): \sigma_{k} \in G\right\} ;$ тогда $\varphi(G) \geqslant \varphi_{0}(G)$, причем согласно стохастической оценке (5) из [5] для $\varphi_{0}(G)$ так же вьполнено

$$
\lim _{G \rightarrow \infty} \mathrm{P}\left(\frac{\varphi_{0}(G)}{u(\lambda\langle G\rangle)} \leqslant x\right)=\Phi_{\alpha}(x) .
$$

Из (12) и (13) получаем утверждение теоремы 3.

Ранеев [5] был получен аналогичньй результат для более частного случая, с использованием других методов.

Автор благодарен А. М. Зубкову за плодотворную идею и А. В. Булинскому за полезное обсуждение.

\section{СПИСОК ЦИТИРОВАННОЙ ЛИТЕРАТУРЫ}

[1] Ивченко Г. И. Вариационный ряд для схемы суммирования независимых величин // Теор. вероятностей и ее примен. 1973. Т. 18. №3. С. 557-570.

[2] Лебедев А. В. Предельные теоремы о максимумах независимых случайных сумм // Теор. вероятностей и ее примен. 1999. Т. 44. №3. С. 631-633.

[3] Kang S., Serfozo R.F. Extreme values of phase-type and mixed random variables with parallel-processing examples // J. Appl. Probab. 1999. V. 36. №1. P. 194-210.

[4] Питербарг В.И., Козлов А. М. О больших скачках случайного блуждания с условием Крамера // Теор. вероятностей и ее примен. 2002. Т. 47. № 4. С. 803-814.

[5] Лебедев А. В. Максимумы субэкспоненциальных полей дробового шума с конечным радиусом влияния // Матем. заметки. 2003. Т. 73. № 2. С. 258-262.

[6] Феллер В. Введение в теорию вероятностей и ее приложения. Т. 2. М.: Мир, 1984.

[7] Лидбеттер М., Линдгрен Г., Ротсен Х. Экстремумы случайных последовательностей и процессов. М.: Мир, 1989.

[8] Рюэль Д. Статистическая механика. М.: Мир, 1971.

[9] Лебедев А.В. Экстремумы полей дробового шума // Фундамент. и прикл. матем. 2001. T. 7. № 4. C. 1081-1090. 